\title{
A Survey on FM-UWB Transceivers
}

\author{
Mohamed Ali and Heba Shawkey \\ Microelectronics Department \\ Electronics Research Institute (ERI) \\ Giza, Egypt
}

\author{
Abdelhalim Zekry \\ Communications and Electronics Department \\ Ain Shams University \\ Cairo, Egypt
}

\begin{abstract}
This paper surveys the research on frequency modulated ultrawideband (FM-UWB) transceivers. FM-UWB system uses low modulation index digital FSK followed by high modulation index analog FM to generate a constant envelope UWB signal with a flat power spectral density and steep spectral roll-off. FM-UWB can be seen as an analog implementation of a spread spectrum system with spreading gain equal to the modulation index. FM-UWB system is suitable for low data rate and short-range applications. The advantages of FM-UWB system such as low power consumption, very low radiated power $(-41.3 \mathrm{dBm} / \mathrm{MHz})$, good coexistence with other existing wireless technologies, and robustness to interference and multipath making it suitable for Wireless Body Area Network (WBAN) in medical applications.
\end{abstract}

\section{General Terms}

Ultra-wideband (UWB), UWB transceivers.

\section{Keywords}

Ultra-wideband (UWB), FM-UWB, Wireless Body Area Network (WBAN).

\section{INTRODUCTION}

Ultra-wideband (UWB) is a promising technology for shortrange and low data rate wireless communications, especially for wireless personal area network (WPAN) and wireless body area network (WBAN) systems. This tremendous growth began after the release for unlicensed uses of the UWB applications within the 3.1-10.6 GHz frequency band by Federal Communications Commission (FCC) in 2002 [1].

Two UWB techniques are considered for short range and low cost UWB systems; impulse radio UWB (IR-UWB) and frequency modulated UWB (FM-UWB). In the IR-UWB system, a sequence of extremely short pulses is transmitted through an antenna [2]. Thus, IR-UWB is a carrier-less transmission technique. However, because of using low duty pulses, synchronization between the transmitter and the receiver becomes very challenging, which increases hardware complexity and power consumption.

On the other hand, the FM-UWB system generates a constantenvelope UWB signal with wideband FM modulation, featuring a very steep spectral roll off [3], [4]. Since the FMUWB receiver can perform FM demodulation without a local oscillator, carrier synchronization is not needed as in the case of the IR-UWB. This makes the overall system design simple and robust. The FM-UWB system is suitable for short-range ( $<10$ meters), low and medium data rate applications (up to $250 \mathrm{kbps})$.

The advantages of FM-UWB system such as low power consumption, very low radiated power $(-41.3 \mathrm{dBm} / \mathrm{MHz})$, good coexistence with other existing wireless technologies, and robustness to interference and multipath making it suitable for Wireless Body Area Network (WBAN) in medical applications[5]. Furthermore, the low radiated power of the FM-UWB is safe for human tissue exposure, which enables FM-UWB to be used as both a sensing and a communication standard for biomedical applications, which gives compact implementation in terms of integrated system [6-9].

\section{FM-UWB TRANSMITTER}

FM-UWB is an analog implementation of a spread spectrum system with spreading gain equal to the modulation index. Figure 1 shows the block diagram of the FM-UWB transmitter. It uses low modulation index digital FSK to encode binary data onto a sub-carrier followed by high modulation index analog FM of the RF carrier to create a constant-envelope UWB signal [3].

The power spectral density of a wideband FM signal reflects the probability density function of the sub-carrier signal $\mathrm{m}(\mathrm{t})$ [10]. Triangular subcarrier waveforms have a uniform probability density function and therefore, produce a flat RF spectrum.

The transmitter comprises a subcarrier oscillator in the range of $\mathrm{MHz}$ to generate a triangular signal that is FSK modulated by the transmitted data. The subcarrier signal $\mathrm{m}(\mathrm{t})$ modulates the RF VCO, generating a constant-envelope UWB signal with a flat power spectral density and steep spectral roll-off.

Figure 2 shows the data, the subcarrier and the UWB signals in the time domain for a data transition at $t=0$. Sub-carrier frequency is $1 \mathrm{MHz}$; the center frequency of the UWB signal was scaled down for the sake of clarity.

A multi-user system may use conventional time-division multiple access (TDMA) or RF frequency division multiple access (FDMA) techniques and also subcarrier FDMA, i.e., different users can be distinguished by different subcarrier frequencies.

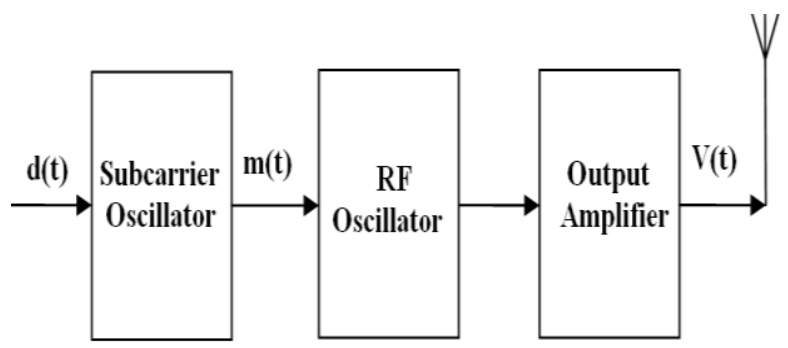

Fig 1: FM-UWB transmitter block diagram 


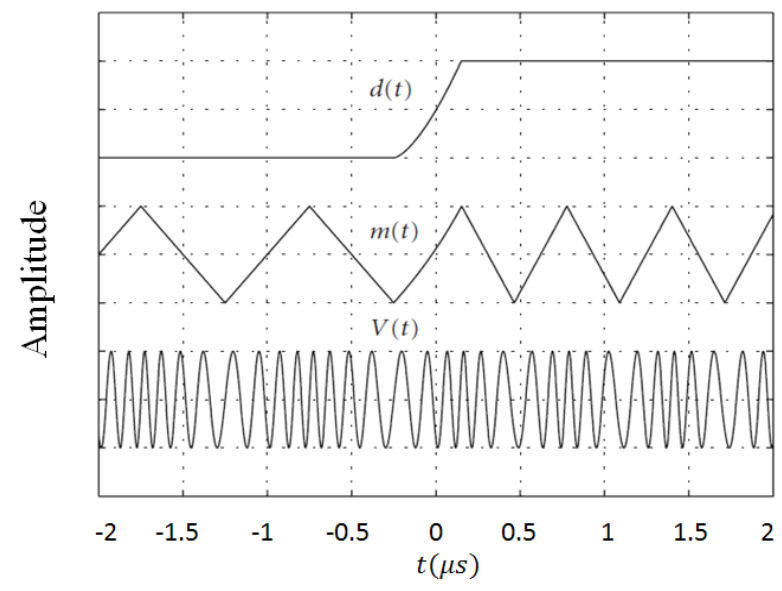

Fig 2: Data d(t), sub-carrier m(t), and UWB signal V(t)

\subsection{Subcarrier Generation}

Direct Digital Frequency Synthesizer (DDFS) is used in the subcarrier generation [11]. In order to provide a digital FSK modulation with high accuracy, a $\Delta \Sigma$ fractional-N PLL is used, instead of using a direct digital frequency synthesizer (DDFS), to generate the subcarrier [12], [13].

For high data rate FM-UWB, >250kbps, the frequency of the subcarrier should be increased. The use of DDFS for high frequency subcarrier generation requires another PLL to generate the high clock frequency. This increases the power consumption of the DDFS. Instead, a finite-modulo fractional$\mathrm{N}$ PLL is used to provide high frequency subcarrier generation [14], [15]. In [16], [17], a successive approximation register frequency-locked loop (SAR-FLL) is used to calibrate the subcarrier frequency which is generated by a relaxation oscillator.

\subsection{RF Carrier Generation}

The most challenging part of the transmitter design is the RF VCO. Many ring VCOs operating at UWB frequencies have been presented in the literature [18-20]. Ring VCO has a wide tuning range and it consumes less silicon area but it suffers from high power consumption and poor phase noise performance. Furthermore, ring VCO is susceptible to supply, temperature, and process variations [21], [22], which make it unsuitable for FM-UWB applications. Hence, LC VCO is a better choice for FM-UWB applications. Furthermore, several LC VCOs operating at the UWB frequencies have been reported [23-31] and are widely used in FM-UWB transmitters for RF carrier generation [12-15], [32-34]. Table 1 provides a comparison among the most recent published VCOs operating at the UWB frequencies. It is clear from the table that the ring VCO consumes more power than the LC $\mathrm{VCO}$ and has poorer phase noise than that of the LC VCO. Also current-controlled ring oscillator (ICO) can be used as the RF source, where the triangular voltage is converted to a current by transconductor, gm [16], [17].

Due to that RF oscillator is open loop modulated and to avoid out of band operation, RF frequency is periodically calibrated. Different techniques have been used to calibrate the RF frequency. A digitally configurable duty-cycled frequencylocked loop has been used in [12].

The RF frequency is quasi-continuously tuned by a $\Delta-\Sigma$ DAC based frequency-locked loop in [13], [15]. In [14], a semidigital quasi-continuous frequency-locked loop (FLL) has been used, which enables digital tuning of the UWB frequency band. A successive approximation register frequency-locked loop (SAR-FLL) has been used to calibrate the RF oscillator frequency in [16], [17]. A single-loop integer-N PLL frequency synthesizer has been used to calibrate the VCO in [32] where multiple lock signals have been used to identify coarse frequency lock and fine phase lock. And to achieve low power requirements, the PLL is only powered up for VCO calibration.

\subsection{The Output Amplifier (OA)}

The output amplifier (OA) drives the antenna and also isolates it from the RF VCO. The OA consumes most of the total transmitter power to maximize the link span between transmitter and receiver. FM-UWB generates a constant envelope RF signal that enables the $\mathrm{OA}$ to operate at its maximum power output where its efficiency is highest [17], [32].

Table 1. VCOs performance comparison

\begin{tabular}{|c|c|c|c|c|c|c|c|}
\hline Ref. & Type & Tech. & VDD (V) & $\begin{array}{c}\text { Power } \\
(\mathbf{m W})\end{array}$ & $\begin{array}{c}\text { Tuning } \\
\text { Range } \\
(\mathbf{G H z})\end{array}$ & $\begin{array}{c}\text { Phase Noise } \\
\mathbf{d B c / H z @ ~} \mathbf{1 M H z})\end{array}$ & FOM \\
\hline$[18]$ & Ring & $180 \mathrm{~nm}$ CMOS & 1.5 & 9.5 & $2.7-4.1$ & -70 & - \\
\hline$[19]$ & Ring & $130 \mathrm{~nm}$ CMOS & 1.2 & 7.48 & $2.41-7.63$ & -84.87 & -153.3 \\
\hline$[20]$ & Ring & $180 \mathrm{~nm}$ CMOS & 1.8 & 48 & $7.95-8.45$ & -88.24 & - \\
\hline$[23]$ & LC & $90 \mathrm{~nm}$ CMOS & 1.6 & 14 & $4.5-7.1$ & -108.5 & -172 \\
\hline$[24]$ & LC & $130 \mathrm{~nm}$ CMOS & 1.8 & 7.8 & $3.31-4.83$ & -120.7 & -185.5 \\
\hline$[25]$ & LC & $90 \mathrm{~nm}$ CMOS & 1.2 & 7.67 & $8.11-15.38$ & -103.9 & - \\
\hline$[26]$ & LC & In GaP/GaAs & 16 & 228.3 & $5.6-16.8$ & -112 & -200 \\
\hline$[27]$ & LC & $130 \mathrm{~nm}$ CMOS & 1.5 & 1.32 & $3.841-5.381$ & -120.63 & -192.13 \\
\hline$[28]$ & LC & $180 \mathrm{~nm}$ CMOS & 1.8 & 6.4 & $4.9-5.46$ & $-100.3 @ 100 \mathrm{~K}$ & -187 \\
\hline$[29]$ & LC & $180 \mathrm{~nm}$ CMOS & 0.8 & 3.92 & $4.567-5.832$ & -116.708 & -183.3 \\
\hline$[30]$ & LC & $180 \mathrm{~nm}$ CMOS & 1.8 & 5.9 & $3.47-5.46$ & -119 & -187.4 \\
\hline$[31]$ & LC & $130 \mathrm{~nm}$ CMOS & 1.2 & 5 & $3.8-5.6$ & -119.21 & -184.9 \\
\hline
\end{tabular}




\section{FM-UWB RECEIVER}

Figure 3 shows the block diagram of the FM-UWB receiver. The receiver in its basic form consists of a wideband FM demodulator, one or several low frequency subcarrier filtering and amplification stages, and subcarrier demodulators [3]. The receiver demodulates the FM-UWB signal without frequency translation. No local oscillator and no carrier synchronization are needed where the receiver synchronization is only limited by the bit synchronization time. This reduces the power and the cost of the receiver.

As the low-noise amplifier (LNA) is the first block of the FMUWB receiver, it should provide enough gain to improve the input signal to noise ratio (SNR) within the power-budget. Several LNA topologies for UWB applications have been presented [35-43].

\subsection{Wideband FM Demodulator}

The key receiver building block is the wideband FM demodulator. A wideband FM demodulator is implemented as a delay-line demodulator that is shown in Figure 4 [15], [34], [44-46]. It transforms the FM to a phase-modulated (PM) signal via a delay line, and then multiplies it with the original FM signal through a multiplier [47] to produce an amplitudemodulated (AM) output suitable for envelope detection. A fully differential delay line RF demodulator has been presented in [48], [49].

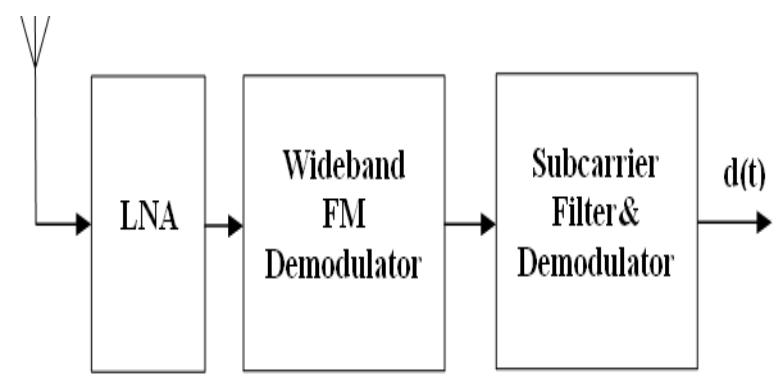

Fig 3: FM-UWB receiver block diagram

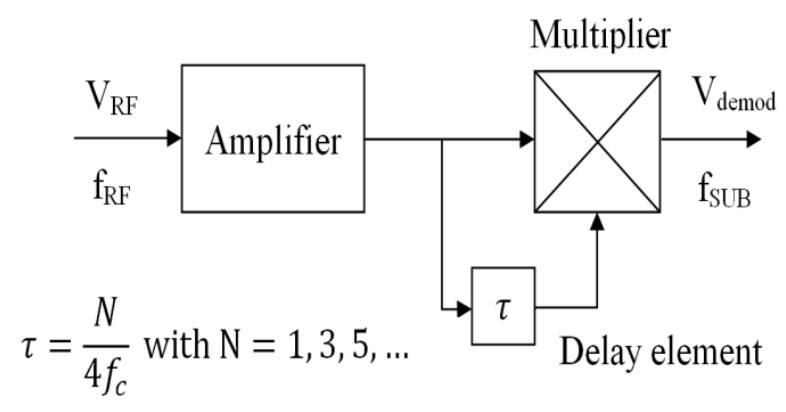

Fig 4: Wideband FM delay line demodulator

The circuits that are used to implement the delay line such as parallel resonant circuit [34], all-pass filter (APF), or the band-pass filter (BPF) [45] employ many inductors, which increase the chip area significantly.

A phase-interpolator based delay line has been proposed in [15]. It is an inductor-less delay circuit. A different demodulation technique has been used in [50], [51] where the FM-AM transformation is realized directly via a bandpass filter. One disadvantage of this technique is that a receiver calibration is required to optimize the SNR for a selected subband. Despite all the aforementioned implementations, FM-
UWB transceivers still meet many design challenges and represent a promising topic for researchers.

\section{CONCLUSIONS}

FM-UWB system generates a constant-envelope UWB signal with wideband FM modulation, featuring a very steep spectral roll off. Since the FM-UWB receiver can perform FM demodulation without a local oscillator, carrier synchronization is not needed. This makes the overall system design simple and robust. FM-UWB system is suitable for low data rate, short-range applications. It is a low complex, low cost, and low power system

\section{REFERENCES}

[1] Federal Communications Commission, "FCC notice of proposed rule making, revision of part 15 of the commission's rules regarding ultra-wideband transmission system", FCC, Washington DC, ET-docket 98-153.

[2] M. Z. Win and R. A. Scholtz, "Impulse radio: How it works?", IEEE Commun. Lett. vol. 2, pp. 36-38, Feb. 1998.

[3] J. F. M. Gerrits, M. H. L. Kouwenhouven, P. R. van der Meer, J. R. Farserotu, and J. R. Long, "Principles and limitations of ultra-wideband FM communications systems", EURASIP J. of App. Signal Proc., vol. 2005:3, pp. 382-396.

[4] J. F. M. Gerrits, J. R. Farserotu and J. R. Long, "LowComplexity Ultra Wideband Communications", IEEE Transactions on Circuits and Systems-II, vol. 55, pp. 329 - 333, Apr. 2008

[5] M. Hernandez and R. Kohno, "UWB Systems for Body Area Networks in IEEE 802.15.6", IEEE International Conference on Ultra-Wideband (ICUWB), 2011.

[6] B. Gupta, D. Valente, E. Cinaca, and R. Prasad, "FMUWB for radar and communications in medical applications", First International Symposium on Applied Sciences on Biomedical and Communication Technologies, 2008. ISABEL '08. , 16 December, 2008.

[7] E. Cinaca and B. Gupta, "FM-UWB for Communications and Radar in Medical Applications", Wireless Personal Communication Journal, July 2009.

[8] W. Rhee, N. Xu, B. Zhou, and Z. Wang, "Low Power Non Invasive UWB Systems for WBAN and Biomedical Applications", International Conference on Information and Communication Technology Convergence (ICTC), 2010, pp. 35-40.

[9] B. Gupta, E. Cianca, M. Ruggieri, and R. Prasad, "End to End Vital Sign Monitoring System with FM-UWB Technology", International Conference on Devices and Communications, 2011.

[10] H. Taub, D. Schilling, Principles of Communication Systems, McGraw-Hill, New York, NY, USA, 1971.

[11] P. Nilsson, J. Gerrits, and J. Yuan, "A low complexity DDS IC for FMUWB applications", 16th IST Mobile and Wireless Communications Summit, Budapest, Hungary, Jul. 2007.

[12] B. Zhou, W. Rhee, and Z. Wang, "Reconfigurable FMUWB transmitter", Electronics Letters, Vol. 47, No. 10 12th May 2011. 
[13] B. Zhou, R. He, J. Qiao, J. Liu, W. Rhee, and Z. Wang, "A Low Data Rate FM-UWB Transmitter with $\Delta-\Sigma$ Based Sub-Carrier Modulation and Quasi- Continuous Frequency-Locked Loop", IEEE Asian Solid-State Circuits Conference, Nov. 2010.

[14] B. Zhou, H. Lv, M. Wang, J. Liu, W. Rhee, Y. Li, D. Kim, and Z. Wang, "A $1 \mathrm{Mb} / \mathrm{s} \quad 3.2-4.4 \mathrm{GHz}$ Reconfigurable FM-UWB Transmitter in $0.18 \mu \mathrm{m}$ CMOS", IEEE Radio Frequency Integrated Circuits Symposium (RFIC), 2011.

[15] B. Zhou, J. Qiao, R. He, J. Liu, W. Zhang, H. Lv, W. Rhee, Y. Li, and Z. Wang, "A Gated FM-UWB System With Data-Driven Front-End Power Control", IEEE Trans. Circuits and Systems I, Regular Papers, Vol. 59, No. 6, June 2012.

[16] N. Saputra, J. R. Long, and J. J. Pekarik, "A 900 $\mu$ W, 3-5 $\mathrm{GHz}$ integrated FM-UWB transmitter in $90 \mathrm{~nm}$ CMOS", IEEE ESSCIRC, Sep. 2010, pp. 398-401.

[17] N. Saputra and J. R. Long, "A fully-integrated, shortrange, low data rate FM-UWB transmitter in $90 \mathrm{~nm}$ CMOS", IEEE J. Solid-State Circuits, vol. 46, pp. 16271635, Jul. 2011.

[18] T. Tong, Z.Wenhua, J. Mikkelsen, and T. Larsen, "A $0.18 \mu \mathrm{m}$ CMOS low power ring VCO with $1 \mathrm{GHz}$ tuning range for 3-5 GHz FM-UWB applications", 10th IEEE Int. Conf. Commun. Syst., 2006, pp. 1-5.

[19] N. Rashidi, A. Nabavi, and M. Mehrabian, "7GHz Voltage Control Ring Oscillator for UWB Applications", International Conference on Microelectronics (ICM), 2008 .

[20] J. Duan, Z. He, C. Kang, J. Wang, and J. Zhang, "A Multiloop Ring VCO Design in $0.18 \mu \mathrm{m}$ CMOS Technology", 10th IEEE International Conference on Solid-State and Integrated Circuit Technology(ICSICT), 2010, pp.99-101.

[21] A. Abidi, "Phase noise and Jitter in CMOS ring Oscillators", IEEE Journal of Solid State Circuits, August 2008.

[22] M. Danesh and J. R. Long, "Ultra-low Power Transmitters for UWB-FM Sensor Networks", 19th annual work shop on circuits, systems and signal processing (ProRisc) Nov.2008, pp. 166-170.

[23] B. Soltanian, H. Ainspan, W. Rhee, D. Friedman, and P. R. Kinget, "An ultra-compact differentially tuned $6-\mathrm{GHz}$ CMOS LC-VCO with dynamic common-mode feedback", IEEE J. Solid-State Circuit, 2007, 42, (8), pp. $1635-1641$.

[24] S. S. Broussev, T. A. Lehtonen, and N. T. Tchamov, "A Wideband Low Phase-Noise LC-VCO With Programmable KVCO", IEEE Microwave and Wireless components Letters, vol. 17, No. 4, April 2007.

[25] M. Demirkan, S.P. Bruss, and R. R. Spencer, "Design of Wide Tuning- Range CMOS VCOs Using Switched Coupled-Inductors", IEEE J. Solid-State Circuits, vol. 43, No.5, May 2008.

[26] M. Tsuru, et al., "A Triple-Tuned Ultra-Wideband VCO", IEEE Transactions on MTT, vol. 56, No. 2, February 2008.
[27] A. EI Oualkadi, "5-GHz Low Phase Noise CMOS LCVCO with PGS Inductor Suitable for Ultra-Low Power Applications", Mediterrannean Microwave Symposium (MMS), 2009.

[28] J. Hou and Y. Wang, "A $5 \mathrm{GHz}$ differential Colpitts CMOS VCO using the bottom PMOS cross-coupled current source", IEEE Microw. Wirel. Compon. Lett., 2009, 19, (6), pp. 4101-403.

[29] C. Wu and G. Jian, "A CMOS LC VCO with novel negative impedance design for wide-band operation", IEEE Radio Frequency Integrated Circuits Symp., (RFIC), Anaheim, CA, USA, 2010, pp. 537-540.

[30] X. Qi and Z. Li, "A Low Power Consumption, Low Phase Noise, and Wide Tuning Range LC VCO with ACC", IEEE 13th International Conference on Communication Technology, (ICCT), 2011.

[31] C.H. Chun, H.S. Choi, Q.D. Bui, S.Y. Kang, J.Y. Jang, U.B. Lee, I.Y. Oh, and C.S. Park, "Compact wideband LC VCO with active inductor harmonic filtering technique", Electronics Letters, Vol. 47 , Issue 3, 2011.

[32] M. Detratti, E. Perez, J. F. M. Gerrits, and M. Lobeira, "A 4.6 mW 6.25-8.25 GHz RF transmitter IC for FMUWB applications", ICUWB, 2009, pp. 180-184.

[33] J. F.M. Gerrits, M. Danesh, Y. Zhao, Y. Dong, G. van Veenendaal, J. R. Long, and J. R. Farserotu, "System and Circuit Considerations for Low-Complexity ConstantEnvelope FM-UWB", IEEE International Symposium on Circuits and Systems (ISCAS), 2010, pp. 3300-3303.

[34] J. F. M. Gerrits et al., "A 7.2 GHz -7.7 GHz FM-UWB transceiver prototype", IEEE ICUWB, Sep. 2009, pp. $580-585$.

[35] C. P. Chang and H. R. Chuang, "0.18 $\mu \mathrm{m} \mathrm{3-6} \mathrm{GHz}$ CMOS broadband LNA for UWB radio", electronics letters, 9th June 2005, Vol. 41 No. 12.

[36] T. Taris, JB. Begueret, and Y. Deval, "A Low Voltage Current Reuse LNA in a 130nm CMOS Technology for UWB Applications", 37th European Microwave Conference, 2007.

[37] D. Hun Shin, J. Park, and C. Patrick Yue, "A LowPower, 3-5-GHz CMOS UWB LNA Using Transformer Matching Techniquev", IEEE Asian Solid-State Circuits Conference, 2007.

[38] M. Battista, J. Gaubert, M. Egels, S. Bourdel, and H Barthélémy, "High-Voltage-Gain CMOS LNA For 68.5-GHz UWB Receivers", IEEE trans. on circuits and systems-II: express briefs, Vol. 55, No. 8, august 2008.

[39] A. Mirvakili, and M. Yavari, "A linear wideband CMOS LNA for 3-5 GHZ UWB Systems", International SoC Design Conference, ISOCC'08, 2008.

[40] Y. Zhao, G. van Veenendaal, H. Bonakdar, J. F.M. Gerrits, and J. R. Long, "3.6mW, 30dB Gain Preamplifiers for an FM-UWB Receiver", IEEE Bipolar/BiCMOS Circuits and Technology Meeting, BCTM 2008, pp. 216-219.

[41] W. Wu, M. Nagaraju, C. T. Charles, and X. Fan, "A 3.1 $5 \mathrm{GHz}$ High and Flat Gain UWB LNA", 3rd IEEE International Symposium on Microwave, Antenna, Propagation and EMC Technologies for Wireless Communications, 2009, pp. 1138-1141. 
[42] A. S. Nilsaz, M. K. Parashkoh, H. g. zadeh, Zhuo, Zou, M. B. Nejad, and L. Rong Zheng, "Low Power $0.18 \mu \mathrm{m}$ CMOS Ultra Wideband Inductor-less LNA Design for UWB Receiver", IEEE Asia Pacific Conference on Circuits and Systems (APCCAS), 2010, pp. 855-858.

[43] E. Abiri, M.R. Salehi, and H. Rezaei, "Design of UWB LNA with Interference Rejection using Coupled Inductors", IEEE Symposium on Industrial Electronics and Applications (ISIEA), Sep. 2011.

[44] J. F. M. Gerrits, J. R. Farserotu, and J. R. Long, "A wideband FM demodulator for a low-complexity FMUWB receiver", 9th Eur. Wireless Technol. Conf., Sep. 2006, pp. 99-102.

[45] Y. Dong, Y. Zhao, J.F.M. Gerrits, G. van Veenendaal, and J.R.Long, "A 9mW High Band FM-UWB Receiver Frontend", Proceeding of ESSCIRC, pp.302-305, Sep. 2008.

[46] Y. Zhao, Y. Dong, J. Gerrits, G. V. Veenendaal, J. Long, and J. Farserotu, "A short range, low data rate, $7.2 \mathrm{GHz}-$ 7.7 GHz FM-UWB receiver front-end", IEEE J. SolidState Circuits, vol. 44, pp. 1872-1882, Jul. 2009.
[47] T. Tong, C. A. Lin, O. K. Jensen, J. H. Mikkelsen, and T. Larsen, "A $0.25 \mu \mathrm{m}$ CMOS Low Power RF Multiplier for Ultra-wide Band System Applications", IEEE Conference on Electron Devices and Solid-State Circuits, 2005 , pp. $221-224$.

[48] T. Tong, J. H. Mikkelsen, T. Larsen, "A $0.18 \mu \mathrm{m}$ CMOS Implementation of a Low Power, Fully Differential RF Front-End for FM-UWB Based P-PAN Receivers", 10th IEEE Singapore International Conference on Communication systems, ICCS, 2006.

[49] T. Tong, J. H. Mikkelsen, T. Larsen, "0.18 $\mu \mathrm{m}$ CMOS RF Front-End Chipset for FM-UWB Based P-PAN Receivers", Norchip, 2007.

[50] N. Saputra, J. R. Long, and J. J. Pekarik, "A $2.2 \mathrm{~mW}$ Regenerative FM-UWB Receiver in 65 nm CMOS", IEEE Radio Frequency Integrated Circuits Symposium, 2010.

[51] N. Saputra and J. R. Long, "A short-range low data-rate regenerative FM-UWB receiver", IEEE Trans. Microw. Theory Tech., vol. 59, pp. 1131-1140, Apr. 2011. 